\title{
О ВОЗМОЖНОСТИ СЕГНЕТОЭЛЕКТРИЧЕСКОГО ФАЗОВОГО ПЕРЕХОДА, ОБУСЛОВЛЕННОГО ПРИМЕСНЫМИ НОСИТЕЛЯМИ
}

В рамках вибронной теории показана возможность возникновения сегнетоэлектрического фазового перехода, обусловленного примесными носителями. Электрон-фононное взаимодействие между примесной и основной зонами индуцирует низкосимметричное искажение решетки при выполнении определенного условия. Существует пороговая концентрация примесных урсвней для возникновения фазового перехода. Рассчитаны температурные зависимости частот мяпких мод, низкосимметричного искажения решепки (поляризации). Найдена формула для точки Кюри.

В [ $\left.{ }^{1}\right]$ рассмотрено влияние неравновесных носителей на точку Кюри широкощельного сегнетоэлектрика в рамках вибронной теории (см., напр., $\left.\left[{ }^{2}\right]\right)$. Показано, что генерация неравновесных носителей приводит к эффективному ослаблению электрон-фононного (вибронного) взаимодействия, ответственного за фазовый переход, и, как следствие, к понижению точки Кюри. Согласно $\left[{ }^{3}\right]$, примесные носители действуют аналогичным образом. На обнаружение такого понижения точки Кюри в SbSI указывается в [ $\left.{ }^{4}\right]$ *

В $\left[{ }^{3}\right]$ рассматривался случай, когда концентрация примесных уровней достаточно мала** и активные предельные оптические колебания не смешивают примесные состояния с зонными; т. е. соответствующее электрон-фононное взаимодействие либо отсутствует из-за соображений симметрии, либо мало по сравнению с определяющим фазовый переход межзонным вибронным взаимодействием.

Изучим теперь случай, когда узкая примесная зона смешивается электрон-фононным взаимодействием с основной узкой зоной кристалла. Перенормированный таким межзонным вибронным взаимодействием электронный спектр системы при этом можно записать в виде $\left[{ }^{7}\right]$

$$
\overline{\varepsilon_{1,2}}=\frac{\Delta}{2} \mp\left[\left(\frac{\Delta}{2}\right)^{2}+\frac{V^{2}}{N_{0}} y^{2}\right]^{1 / 2},
$$

где $\Delta$ - затравочная щель между указанными зонами (влияние дисперсии можно приближенно учесть перенормировкой $\left.\Delta\left[{ }^{8}\right]\right) ; V-$ константа межзонного вибронного взаимодействия, отвечающего в данном

\footnotetext{
* В [5] обнаружено существенное влияние примесных носителей на точку Кюри $\mathrm{SnTe}$.

** Концентрационные зависимости жонстанты Кюри-Вейсса и точки КюриВейсса сегнетоэлектрических твердых растворов типа (Ba, $\mathrm{Sr}$ ) $\mathrm{TiO}_{3}$ рассматриваются B $\left[{ }^{6}\right]$.
} 
случае *** активному колебанию с нормальной координатой $y ; N_{0}$ число элементарных ячеек в кристалле.

Константы такого электрон-фононного взаимодействия $V$ могут иметь значительную величину, когда взаимодействие резонансное ( $\omega_{\omega} \sim \Delta, \omega-$ затравочная частота активного колебания).

Предполагается, что число перенормированных вибронным взаимодействием состояний в основной зоне равно числу состояний в примесной зоне, т. е. учитываются только интегралы перекрывания между активными в вибронном взаимодействии состояниями, которые относятся к одной ячейке.

Аналогично $\left[{ }^{7}\right]$ на основе (1) получаем выражение для свободной энергии активной в фазовом переходе электрон-фононной подсистемы:

$$
\begin{gathered}
F(T, y)=\sum_{\sigma=1,2,3} n_{\sigma} \mu-N_{d} k_{\mathrm{B}} T \ln \left[\left(1+\mathrm{e}^{\left[\mu-\frac{\Delta}{2}+\hat{\Delta}(y)\right] \frac{1}{k_{\mathrm{B}} T}}\right) \times\right. \\
\left.\times\left(1+\mathrm{e}^{\left[\mu-\frac{\Delta}{2}-\hat{\Delta}(y)\right] \frac{1}{k_{\mathrm{B}} T}}\right)\right]-\left(N-N_{d}\right) k_{\mathrm{B}} T \ln (1+ \\
+\mathrm{e}^{\left.(\mu-\Delta) \frac{1}{k_{\mathrm{B}} T}\right)}+\frac{1}{2} M \omega^{2} y^{2},
\end{gathered}
$$

где

$$
\begin{gathered}
\hat{\Delta}(y)=\left(\frac{\Delta^{2}}{4}+\frac{V^{2}}{N_{0}} y^{2}\right)^{1 / 2}, \\
n_{1,2}=N_{d}\left[\mathrm{e}^{\left.\left[\frac{\Delta}{2}-\mu \mp \hat{\Delta}(y)\right] \frac{1}{k_{\mathrm{B}} T}+1\right]^{-1}=N_{d} f_{1,2},}\right. \\
n_{3}=\left(N-N_{d}\right)\left[\mathrm{e}^{\left.(\Delta-\mu) \frac{1}{k_{\mathrm{B}} T}+1\right]^{-1}=\left(N-N_{d}\right) f_{3} .}\right.
\end{gathered}
$$

Здесь $\mu$ - химический потенциал системы; $N_{d}$ - число доноров (считается, что при $T=0$ донорная примесная электронная зона **** заполнена); $N$ - число состояний в зоне проводимости; $f_{\sigma}-$ числа заполнения зон, причем индекс $\sigma=1$ соответствует донорной зоне, а $\sigma=2,3-$ расщепленной электрон-фононным взаимодействием зоне проводимости; $M$ - приведенная масса, отвечающая активному колебанию.

Химический потенциал $\mu$ определяется из условия $\frac{\partial F}{\partial \mu}=0$, которое, согласно (2), запишется в виде ******

$$
\sum_{\sigma=1,2,3} n_{\sigma}=N_{d}
$$

*** Из-за вырождения в k-пространспве из зонных состояний можна построить волновые функции точечной симметрии, которую должен иметь данный уровень. Поэтому возможно смешивание основной и примесной зон нечетным колебанием (из соображений симметрии).

**** Случай, когда основной зоной является валентная, а примесной - акцепторная, аналотичен.

***** Счнтается, что щель между валентной зоной и зоной проводимости велика, так что соответствующие тепловые перебросы электронов отсутствуют, 

$\frac{\partial F}{\partial y / y_{0}}=0$. Оно определяется уравнением

Низкосимметричное искажение решетки находим из условия

$$
\hat{\Delta}\left(y_{0}\right)=\frac{1}{2} \tau(x) \Delta\left\{\frac{\operatorname{sh} \frac{\hat{\Delta}\left(y_{0}\right)}{k_{\mathrm{B}} T}}{\operatorname{ch}\left[\left(\frac{\Delta}{2}-\mu\right) \cdot \frac{1}{k_{\mathrm{B}} T}\right]+\operatorname{ch} \frac{\hat{\Delta}\left(y_{0}\right)}{k_{\mathrm{B}} T}}\right\},
$$

где

$$
\tau(x) \equiv \frac{2 \bar{V}^{2} x}{M \omega^{2} \Delta}, \quad \bar{V} \equiv V \sqrt{\frac{N}{N_{0}}}, \quad x \equiv \frac{N_{d}}{N},
$$

а $\hat{\Delta}\left(y_{0}\right)$ дано согласно формуле (3).

Из уравнений (3) и (6) следует, что температурная зависимость искажения решетки $y_{0}$ обусловлена изменением равновесного заполнения электронами примесной и основной зон.

При $T=0$ квадрат низкосимметричного искажения решетки равен

$$
\frac{y_{0}^{2}(T=0)}{N_{0}}=\frac{\Delta^{2}}{4 V^{2}}\left[\tau^{2}(x)-1\right],
$$

т. е., как видно, для существования искажения $y_{0}(T=0)$ необходимо выполнение условия

$$
\tau(x)>1 \text {. }
$$

Отсюда вытекает, что искажение решетки $y_{0}(T=0)$ возникает только начиная с определенной концентрации доноров и при достаточно сильном вибронном взаимодействии $\bar{V}$. Кроме того, существованию низкосимметричной фазы благоприятствует малость как щели $\Delta$, так и частоты $\omega$ затравочного колебания.

Для нахождения температурной зависимости $y_{0}(T)$ система уравнений (5) и (6) решалась численно. Поведение $y_{0}$ с температурой иллюстрирует рис. 1 (параметры: $\bar{V}=1$ э $B / \AA, M \omega^{2}=0,4$ э $B / \AA^{2}$, $\Delta=0,05$ э $B, N=2 N_{0}, x=0,05$; в дальнейшем используются те же параметры).

Точка Кюри структурного (сегнетоэлектрического) фазового перехода, определяемая из условия $\frac{y_{0}\left(T_{\mathrm{c}}\right)}{\sqrt{N_{0}}}=0$, равняется

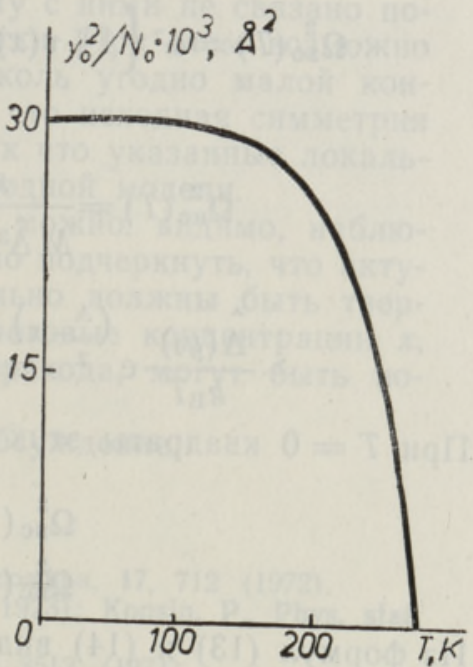

Рис. 1. Зависимость квадрата низкосимметричного искажения $y_{0}^{2} / N_{0}$ от температуры.

$$
k_{\mathrm{B}} T_{\mathrm{c}}=\Delta\left\{\ln \frac{1}{4 x}\left[\left[\frac{(1+x)(\tau(x)+1)}{\tau(x)-1}\right]^{2}-(1-x)^{2}\right]\right\}^{-1}
$$




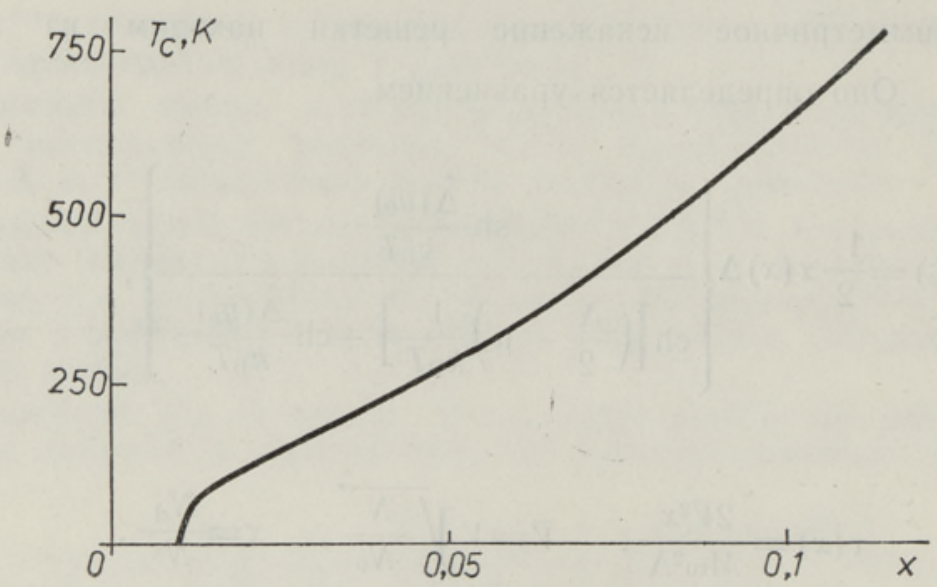

Рис. 2. Зависимость $T_{\text {c }}$ от $x$.

Согласно формуле (10), с ростом $x$ (при прочих фиксированных параметрах) точка Кюри повышается. При $\tau(x) \rightarrow 1$ имеет место $T_{\mathrm{c}} \rightarrow 0$. Зависимость $T_{\text {c }}$ от $x$ показана на рис. 2 , при этом предположено, что $\bar{V}$, $\omega, \Delta$ не изменяются с $x$.

Разлагая $F(T, y)$ в ряд по $y$ около $y_{0}=0$ и $y_{0}(T) \neq 0 \quad\left(\stackrel{2}{y_{0}}(T)>0\right)$, определяемым уравнениями (5) и (6), получаем, аналогично $\left[{ }^{7}\right]$, квадраты частот мягкой моды соответственно в высоко- и низкосимметричной фазах:

$$
\begin{gathered}
\Omega_{\mathrm{BC}}^{2}(T)=\omega^{2}\left\{1+\tau(x)\left[\frac{2(1+x)}{1+x+\sqrt{(1-x)^{2}+4 x \mathrm{e}^{\frac{\Delta}{k_{\mathrm{B}} T}}}}-1\right]\right\}, \\
\Omega_{\mathrm{HC}}^{2}(T)=\frac{x}{M \hat{\Delta}^{3}\left(y_{0}\right)}\left(\frac{\bar{V} V y_{0}}{\sqrt{N_{0}}}\right)^{2}\left\{f_{1}\left(y_{0}\right)-f_{2}\left(y_{0}\right)-\right. \\
-\frac{\hat{\Delta}\left(y_{0}\right)}{k_{\mathrm{B}} T} \mathrm{e}^{\left.\left(\frac{\Delta}{2}-\mu\right) \frac{1}{k_{\mathrm{B}} T}\left[\mathrm{e}^{\frac{\hat{\Delta}\left(y_{0}\right)}{k_{\mathrm{B}} T}} f_{2}^{2}\left(y_{0}\right)+\mathrm{e}^{-\frac{\hat{\Delta}\left(y_{0}\right)}{k_{\mathrm{B}} T}} f_{1}^{2}\left(y_{0}\right)\right]\right\} .}
\end{gathered}
$$

При $T=0$ квадраты этих частот равны

$$
\begin{aligned}
& \Omega_{\mathrm{Bc}}^{2}(T=0)=\omega^{2}[1-\tau(x)], \\
& \Omega_{\mathrm{Hc}}^{2}(T=0)=\omega^{2}\left[1-\tau^{-2}(x)\right] .
\end{aligned}
$$

Из формул (13) и (14) видно, что при $\tau(x)>1$ и $T=0$ высокосимметричная фаза неустойчива, а низкосимметричная фаза стабильна. Температурная зависимость квадрата частоты мягкой моды $M \Omega^{2}$ в обеих фазах изображена на рис. 3.

В системе возникает, вообще говоря, спонтанная поляризация $P_{s}=\frac{\bar{e} y_{0}}{v \sqrt{N_{0}}}$, где $\bar{e}-$ эффективный заряд, отвечающий активной моде, $v-$ объем элементарной ячейки. В случае, когда $\bar{e} \rightarrow 0$, сегнетоэлектрический характер фазового перехода может быть определен из свойств 
Рис. 3. Температурная зависимость квадрата частоты мягкой моды в обеих фазах.

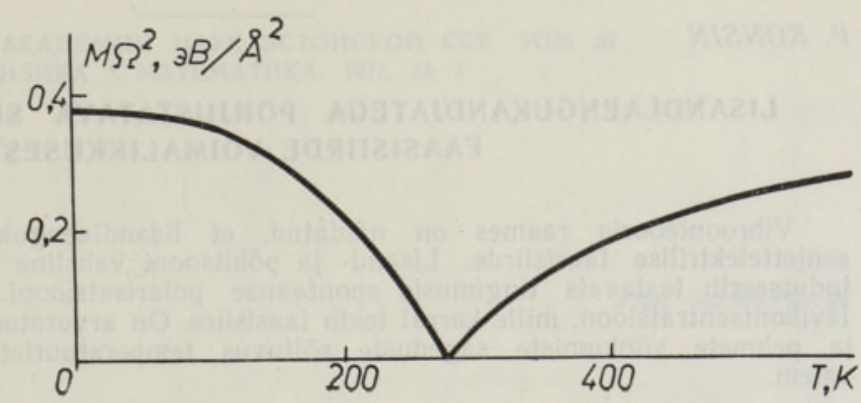

мягкой моды. При этом статическая диэлектрическая восприимчивость равна $\left[{ }^{2}\right] \chi_{\mathrm{Bc}, \mathrm{нc}}=\frac{\bar{e}^{2}}{v M \Omega_{\mathrm{Bc}, \mathrm{Hc}}^{2}}$, где $\Omega_{\mathrm{Bc}, \mathrm{Hc}}^{2}$ определяется выражениями (11) и (12).

Учет собственно-фононной ангармоничности и влияния через межзонное электрон-фононное взаимодействие динамики решетки на электронный энергетический спектр (отклонений от приближения среднего поля) приводит к возможности фазового перехода первого рода $\left[{ }^{8}\right]$ и перенормировке затравочных частот $\omega\left[{ }^{2,8}\right]$.

Случай, когда примесная зона является акцепторной, а основная валентной, аналогичен, только под $N_{d}$ и $N$ следует подразумевать число акцепторных уровней и число состояний в валентной зоне, заполненной при $T=0$ электронами. В частности, зависимости, приведенные на рис. $1-3$, сохраняются.

Конечно, электроны взаимодействуют также с другими колебаниями. Но эти колебания четной симметрии, и поэтому с ними не связано появление низкосимметричного искажения решетки. При этом возможно возникновение локальных деформаций при сколь угодно малой концентрации доноров. Однако здесь полагается, что исходная симметрия системы отвечает наличию примесной зоны, так что указанные локальные искажения неявно фигурируют уже в исходной модели.

Рассматриваемого типа фазовые переходы можно, видимо, наблюдать и в системах типа (Ge, Sn) Tе. Необходимо подчеркнуть, что актуальные в данном случае системы не обязательно должны быть твердыми растворами или сплавами, так как пороговые концентрации $x$, необходимые для возникновения фазового перехода, могут быть порядка $10^{-2}-10^{-3}$.

Автор благодарен Н. Н. Кристофелю за обсуждение.

\section{Л ИТ Е Р А Т Р А}

1. Консин П. И., Кристофель Н. Н., Кристаллография, 17, 712 (1972).

2. Krist of $f$ el, N., Kons in, P., Ferroelectrics, 6, 3 (1973); Konsin, P., Phys. stat. sol. (b), 76, 487 (1976).

3. Кристофель Н. Н. Консин П. И., ФТТ, 13, 3513 (1971).

4. Балецкий Ю. Д., Бе ч а Д. М., Коперлес Б. М., Т уряница И. Д., ФTT, 16, 278 (1974).

5. I izumi, M., H a m a gu chi, Y., Komatsubara, K. F., Kato, Y., J. Phys. Soc. Japan, 38, 443 (1975).

6. Кон син П. И., ФТТ, 16, 2709 (1974).

7. Kristoffel, N., Kons in, P., Phys. stat. sol., 28, 731 (1968).

8. Консин П., Кристофель Н., Изв. АН ЭССР, Физ. Матем., 20, 37 (1971).

$\begin{array}{cc}\text { Ннститут физики } & \text { Поступила в редакцию } \\ \text { Академии наук Эстонской ССР } & 22 / \mathrm{XI} 1976\end{array}$


P. KONSIN

\section{LISANDLAENGUKANDJATEGA PÖHUSTATAVA SENJETTELEKTRILISE FAASISIIRDE VÕIMALIKKUSEST}

Vibroonteooria raames on näidatud, et lisandlaengukandjad võivad põhjustada senjettelektrilise faasisiirde. Lisand- ja põhitsooni vaheline elektron-foononinteraktsioon indutseerib teatavais tingimusis spontaanse polarisatsiooni. Eksisteerib lisandinivoode lävikontsentratsioon, mille korral tekib faasisiire. On arvutatud spontaanse polarisatsiooni ja pehmete võnkumiste sageduste sõltuvus temperatuurist ja saadud Curie punkti valem.

P. KONSIN

\section{ON THE POSSIBILITY OF FERROELECTRIC PHASE TRANSITION CAUSED BY IMPURITY CARRIERS}

In the frames of the vibronic theory it is shown that impurity carriers can cause a ferroelectric phase transition. The electron-phonon interaction between impurity band and host band induces spontaneous polarization at the fulfilment of a definite condition, depending on the concentration of carrier levels. The temperature dependences of spontaneous polarization and soft mode frequencies are calculated. The formulae for the Curie point has been found. 